\title{
Interplay between bladder microbiota and overactive bladder symptom severity: a cross-sectional study
}

\author{
Kun $\mathrm{Li}^{1}$, Chunxiao Chen ${ }^{1}$, Jiarong Zeng ${ }^{2}$, Yuehui Wen ${ }^{1}$, Biao Li ${ }^{1}$, Weihong Chen ${ }^{1}$, Jie \\ Zhao ${ }^{3}$, and Peng $\mathrm{Wu}^{1}$ \\ ${ }^{1}$ Southern Medical University Nanfang Hospital \\ ${ }^{2}$ Meizhou City People's Hospital \\ ${ }^{3}$ Southern Medical University
}

September 11, 2020

\begin{abstract}
Objective To investigate the relationship between the bladder microbiota and overactive bladder (OAB) symptom severity. Design Cross-sectional study of female OAB patients who contributed catheterised urine samples, completed validated symptom questionnaires, and provided demographic data. Setting Nanfang Hospital, Southern Medical University. Population Adult women with overactive bladder symptom. Methods 70 OAB patients were recruited, finished symptoms questionnaires, demographic data, and contributed catheterised urine samples that were analysed by $16 \mathrm{~S}$ rRNA gene sequencing. Main outcome measures Associations between the total/sub scores of Overactive Bladder Symptom Score (OABSS) and bladder microbiota (diversity, overall community structure, and specific organisms). Results We found that bacterial diversity (Simpson index, $P$ $=0.024)$ and richness (Chao1, $P=0.023$ ) of mild patients (OABSS [?]5, $\mathrm{N}=17$ ) were lower than those of moderate/severe patients (OABSS $>5, \mathrm{~N}=53$ ). And bacterial communities of two groups were significantly different. Further, there were positive correlations between scores of OABSS and both richness (Chao1, $P=0.002$ ) and diversity (Shannon index, $P=0.044$ ) of urinary microbiome. Some bacterial genera (e.g., Porphyromona and Prevotella) were significantly related to sub-symptoms of OAB. Conclusion This cross-sectional analysis revealed that increased diversity and richness of the bladder microbiota was associated with worse OAB severity, and specific urinary dysbiosis may constitute an etiological factor in exacerbating functional bladder disorders. Keywords overactive bladder; LUTS; urinary microbiome; urinary microbiota; bladder microbiota Funding National Natural Science Foundation of China (Grant No. 81870522) Tweetable abstract Bladder microbiota is associated with female OAB severity.
\end{abstract}

\section{INTRODUCTION}

With the prevailing dogma that urine is normally sterile has been overturned, ${ }^{1,2}$ there has been growing evidence that a shift in the normal microbiome of the bladder may play an important role in pathophysiology of lower urinary tract symptoms (LUTS) ${ }^{3-7}$ Karstens and Pearce found that bladder urine from women with urge urinary incontinence (UUI) seem to present an altered microbiome compare to urine from asymptomatic healthy individuals, ${ }^{2,5}$ and our previous study found that urinary microbiome may play a part in the development of overactive bladder (OAB) symptom. ${ }^{8}$ However, no research has been published on whether the female urinary microbiome (FUM) affects the severity of $\mathrm{OAB}$ while the effects of $\mathrm{OAB}$ on quality of life increased with the worsening of the disease, ${ }^{9}$ and the factors affecting the severity are unclear. In addition, comprehension of the impact of FUM on the OAB severity may help provide guidance for optimized management, while current guidelines propose symptomatic treatment despite the severity of OAB.

Thus, in order to investigate whether the microbiome/microbiota (e.g., diversity, overall community structure, and/or specific organisms) would play a role in the severity of OAB, we describe the FUM analysis 
using $16 S$ ribosomal RNA (rRNA) gene sequencing to characterize the relationships between FUM parameters and demographic and clinical characteristics of $\mathrm{OAB}$ patients.

\section{MATERIAL AND METHORD}

\section{Subject Recruitment and Urine Collection}

This cross-sectional study began following Institutional Review Board approval. Participants gave verbal and written research consent for chart abstraction and urine collection with analysis for research purposes. Between June 2020 and August 2020, adult patients aged 18 or above, diagnosed with OAB were recruited into this study at Nanfang Hospital in China. Patients must complain of urinary urgency, with or without urgency incontinence, usually with frequency and nocturia, in the absence of infection or other pathological changes, in accordance with the 2010 ICS definition of OAB. ${ }^{10}$ We excluded women who had UTI (based on routine urine test and urine culture) or history of recurrent UTI, antibiotic exposure in the past 4 weeks, immunologic deficiency, neurological bladder, genitourinary cancer, pelvic radiation, urinary stones, untreated symptomatic pelvic organ prolapse (POP) greater than POP-Q stage II, or pregnancy. All participants were required to finish Overactive Bladder Symptom Score (OABSS), and divided into mild and moderate/severe group by total score and the higher the score, the more severe the symptoms of OAB. Scores [?] 5 were defined as mild symptom, and Scores $>5$ were defined as moderate/severe symptom.

Urine was collected using aseptic technique with a sterile catheter by a trained and licensed practitioner. The total volume of urine was emptied from the participant's bladders and was kept at 4, and immediately transferred to laboratory within an hour for centrifugation at 16,000g for $10 \mathrm{~min}$ and the resulting pellet were stored at -80 until further processing.

\section{DNA Isolation, PCR amplification and 16S rRNA Gene Sequencing}

To minimize contamination, DNA extraction was carried out in the laminar flow engine hood using the cell culture scheme provided by the DNasy Blood and Tissue Kit (Qiagen, Germany). The concentration of extracted DNA was determined using a Nanodrop ND-1000 spectrophotometer (Thermo Electron Corporation, USA). The $16 S$ rRNA sequence was amplified by PCR using the primer set for V3-V4 region. Extracted negative controls (no urine) and PCR negative controls (no templates) were included to assess the contribution of extraneous DNA from reagents. The Qiaquick PCR purification kit (Qiagen of Valencia,USA) was used to purify the final PCR product from the nucleotides and primers that had not been incorporated. The purified samples were normalized to the same DNA concentration and sequenced by Illumina MiSeq sequencer (Illumina, USA).

\section{Statistical Analysis}

\section{Clinical Data Analysis}

Continuous variables were reported as means and standard deviations (SD) or medians and interquartile ranges (IQR); categorical variables are reported as frequencies and percentages. Differences in baseline characteristics between cohorts were evaluated using t-tests for continuous variables, Pearson chi-square and Fisher's exact tests were performed for categorical variables. Bivariate correlation analyses were conducted to detect the direction and strength of relations between score of each items in OABSS (e.g., Daytime frequency, Nighttime frequency, Urgency, Urgency incontinence) and bacterial abundance and the relations between clinical data and indices of bacterial alpha diversity using Spearman correlation. Statistical analysis was performed using the Statistical Package for Social Science (SPSS, version 21, USA). For differentially abundant taxa between cohorts, Wilcoxon rank sum test was applied, and Benjamini-Hochberg false discovery rate correction was performed in $\mathrm{R}$ (version 3.4.1, stats package). Statistical tests were based on two-tailed probability and the results were considered significant when the $P$ value was less than 0.05 .

The wrapper package Quantitative Insights Into Microbial Ecology (QIIME) was applied to process the raw reads to create an operational taxonomic units (OTUS) table. Using an open reference selection strategy with Uclust, the sequences were clustered into individual OTU at the default similarity level of $97 \%$, and 
then chimera detection was performed using the the program UCHIME. Using Ribosomal Database Project Classifier to align a single representative sequence from each clustered OTU to the Greengenes database.

Alpha diversity, including the Observed species, Chao1, Shannon, Simpson and Abundance-Based Coverage Estimator (ACE) and Pielou's index, was evaluated using QIIME. The Chao1, ACE and the Observed species were used to calculate richness, samples with larger values are richer. Evenness was calculated with Pielou's Index, which ranks samples from 0 to 1 , with 1 being completely even, while a smaller index score indicates that certain species are more abundant than others. Shannon and Simpson index combines interactions between richness and evenness, Larger Shannon diversity values indicate more diverse communities with greater richness and/or evenness, and Simpson diversity is the opposite. The difference of alpha diversity was evaluated by Wilcoxon rank sum test. Beta-diversity, measured by calculating the Bray Curtis, weighted UniFrac and unweighted UniFrac distances, was used to compare microbial composition between samples. Taxa summaries were reformatted and inputted into Linear discriminant analysis effect size (LEfSe) via the Huttenhower Lab Galaxy Server to identify significantly different bacteria as biomarkers between groups at genus level. In the settings of LEfSe, ${ }^{11}$ the significantly specific bacteria were identified using the MannWhitney U test, and their effect size were estimated via linear discriminant analysis (LDA). The threshold on the logarithmic LDA score for discriminative features was 2.0.

\section{RESULTS}

\section{Participant Demographic Characteristics and Clinical Symptoms}

82 women with $\mathrm{OAB}$ symptoms were recruited. However, twelve samples were excluded due to have a low amount of bacterial DNA below the detection limit. Finally, 70 female patients were divided into mild group ( $\mathrm{M}$ group, $\mathrm{N}=17$ ) and moderate/severe group (M/S group, $\mathrm{N}=53$ ) respectively. All the urine samples from these participants were verified cultured negative during the standard culture procedure. The demographic characteristics between two cohorts, including BMI, history of pelvic surgery, pregnancy and estrogen treatment were similar (Table 1). The mean age of $\mathrm{M}$ group was 37.1 years and of $\mathrm{M} / \mathrm{S}$ group was 38.4 years $(P=0.675)$. Most of patients were currently married ( $\mathrm{M}$ group $88 \%, \mathrm{M} / \mathrm{S}$ group $79 \%$ ) and only a small number of these participants were premenopausal (M group 29\%, M/S group 26\%). Although five patients in the $\mathrm{M} / \mathrm{S}$ group had diabetes, the difference was not statistically significant $(P=0.439)$. Consistent with the entrance requirements for the study, the score of OABSS was significantly higher in moderate/severe than mild participants.

\section{Sequencing Data and Relative Abundances of Urinary Bacteria}

We obtained 3,926,088 sequences, 5,802,274 reads from the 70 samples. The median number of reads in mild group was 77,177 , and in the moderate/severe group was $78,329(P=0.951)$. The reads were classified into 4,415 OTUs that were used for downstream analyses. Venn diagrams demonstrated that 1019 OTUs were shared between the two cohorts (Figure S1). The median number of OTUs was significant increased in $\mathrm{M} / \mathrm{S}$ group (M group 177, M/S group 208, $P=0.043$ ). Most of the OTUs were classified at the genus level and these OTUs belong to 47 different Phyla from all urine specimens. Relative abundance of bacteria was used to detect the composition of bacteria phyla and families (Figure S2, Table 2). The most common phylum in both two groups wasFirmicutes (M group 47.7\%, M/S group 37.0\%), followed by Proteobacteria (M group 28.2\%, M/S group 24.7\%),Actinobacteria (M group 5.1\%, M/S group 12.2\%) andBacteroidetes $(\mathrm{M}$ group $5.0 \%, \mathrm{M} / \mathrm{S}$ group $7.8 \%$ ). And phylumActinobacteriawas significantly more abundant in the moderate/severe group $(P=0.018)$. Bacteria from these four phyla were found in all urine specimens at some level. In general, the most abundant bacterial families with mean abundances $>2 \%$ wereLactobacillaceae ,Veillonellaceae ,Staphylococcaceae, Enterococcaceae andStreptococcaceae(Firmicutesphylum), Moraxellaceae ,Methylobacteriaceae and Sphingomonadaceae(Proteobacteriaphylum),Bifidobacteriaceae(Actinobacteria phylum),Prevotellaceae(Bacteroidetes phylum). Although these families are different between the two groups to some extent, as shown in Table 2, only the family Bifidobacteriaceae was found at significantly higher mean abundances in mild cohort when compared to the moderate/severe cohort. $(P=0.04)$. At the genus level, 7 genera had mean abundances $>2 \%$. These include Lactobacillus andStreptococcus (Firmi- 
cutes phylum), Gardnerella(Actinobacteria phylum), Prevotella (Bacteroidetesphylum), Methylobacterium ,Acinetobacter and Sphingomonas (Proteobacteriaphylum).

\section{Individual Variability}

Urine specimens were classified into urotypes, defined by the predominant ( $>50 \%$ abundance) genus present. ${ }^{2}$ If no genus reached $50 \%$ abundance, the urotype was classified as 'divese'. And a total of 27 urine specimens had matching urotypes, while all other samples were diverse. The most frequent urotype in both cohorts was Lactobacillus (M group 29.4\%, M/S group 22.6\%). Two urotypes were present in both cohorts (Lactobacillus and Streptococcus ). TheHaemophilus, Escherichia-Shigella, Enterococcus andSneathia urotypes were present only in the mild cohort, whereas the Gardnerella urotype was present only in the moderate/severe cohort; however, these differences were not statistically significant at our current sample size. The number of different bacterial genera in each individual's urinary microbiome ranged from 24 to 117 (mild range 24-86 genera per sample, moderate/severe range 30-117 genera per sample).

\section{Alpha Diversity}

Alpha diversity, measured by the Observed species, Chao1, Shannon, Simpson, ACE and Pielou index, was described in the methods. The indices of bacterial alpha diversity were listed in Table 3. The Observed species, Chao1 and ACE (which calculate alpha diversity measures of richness) were much higher in the moderate/severe cohort (Figure 1A, Observed species, $P=0.044$; Figure 1B, Chao1, $P=0.023$; Figure 1C, ACE, $P=0.010$ ). The Shannon index (Figure 1D, $P=0.015$ ) and Simpson (Figure 1E, $P=0.024$ ) illustrated the bacterial diversity was markedly lower in the mild symptoms patients. However, the Pielou index (Figure 1F, $P=0.064$ ) indicated that there was no significant difference in bacterial evenness between the two groups.

The Observed species, Chao1, ACE, and Shannon's Indices were significantly correlated to OABSS of the overall participant population (Figure 2A, B, C, D), and Simpson's Index (Figure 2E) approached significance for this trend; however, there was no correlation with Pielou's values (Figure 2F). Therefore, OAB scores correlated with richness, but not evenness.

\section{Beta Diversity}

Based on unweighted UniFrac, weighted UniFrac, and Bray-Curtis distance metrics, the PCoA was performed to measure the similarity of microbial communities in the two corhorts (Figure 1G, H, I). ANOSIM revealed that there were significant differences in bacterial communities between mild and moderate/severe groups using the Bray-Curtis distance metric (statistic $\mathrm{R}=0.130, P=0.021$, number of permutations $=999$ ). However, this clustering was not significant when the analysis was based on unweighted UniFrac distance metric (statistic $\mathrm{R}=0.044, P=0.22$, number of permutations $=999$ ) and weighted UniFrac distance metric (statistic $\mathrm{R}=0.031, P=0.258$, number of permutations $=999$ ).

\section{Specific Genera Associated with severity and sub-symptoms of OAB}

The LEfSe algorithm was utilized to identify the specific bacteria genera associated with OAB severity, and revealed no significant difference in bacterial genera between two groups. Spearmans correlation analysis was utilized to identify the specific bacteria genera associated with sub-symptoms of OAB. In all, eight genera showed significant correlation with one or more of the OABSS components; one with Daytime frequency, four with Nighttime frequency, two with Urgency, and two with Urgency incontinence as shown in Table 4. The strongest correlation was between Bosea and Nighttime frequency (Correlation coefficient $=0.472, P<$ $0.001)$.

\section{DISCUSSION}

\section{Main findings}

In this cross-section study we demonstrated that Firmicutes was the most common phylum and Lactobacillus was the most common genus in the bladders of adult women with OAB; However, there were significant 
differences in the composition of bladder microflora in patients with different degrees of OAB. The two groups with different severity of $\mathrm{OAB}$ symptom have different urotypes. In addition, with the increase of the severity of $\mathrm{OAB}$, the diversity and richness of bladder flora increased. And several specific genera, including Flavobacterium ,Bosea, Campylobacter, Porphyromonas ,Prevotella, Ezakiella, Bacillus and Massiliaare associated with different $\mathrm{OAB}$ sub-symptoms. These findings suggest that the diversity, presence, or absence of certain bacteria may play a key role in the pathophysiology of OAB.

\section{Strengths and limitations}

This study has several major strengths. First, we used catheterized urine specimens instead of voided urine. Indeed, Wolfe and coworkers discovered that the microbiomes in voided urine and urine collected by transurethral catheter differed, and microbiome in catheterized urine specimens most closely represent the bladder microbiome itself, without contribution of microbial flora from the genital tract. ${ }^{12}$ Second, except the scores of OABSS, the demographic characteristics (e.g., age, BMI, race/ethnicity) of the two cohorts were similar. Therefore, many factors that cause the heterogeneity of flora are excluded. Third, the total sample size of well-characterised $\mathrm{OAB}$ patients included in this study is relatively large. In addition, all patients not only completed the routine urine examination, but also underwent standard urine culture to rule out urinary tract infection. The limitations of the study include small sample size of mild OAB group and lack of a replication dataset. And it is hardly to determine the cause-effect relationship between symptoms and bioinformatics indicators in this cross-sectional study. Thus, prospective studies with a larger sample will be required to clarify the role of urinary microbiota in the development and progression of OAB.

\section{Interpretation}

Since the detection of microorganisms in the bladder of female without UTI, ${ }^{12}$ additional confirmatory evidence has clearly demonstrated the presence of a living bacterial community in the bladder of both women with and without lower urinary tract symptoms. ${ }^{2,}$, $13-15$ This significant paradigm shift raises many questions about the role of the female urinary microflora in lower urinary tract health and disease. In this cross-section study, we characterized the differences of urinary microbiomes between the women with different degrees of OAB symptoms using high throughput sequencing of the bacterial $16 S$ rRNA gene.

Besides diverse, the most common urotype in both groups was Lactobacillus, and similar results were observed by PRICE in UUI patients. ${ }^{16}$ In addition, we found mild group had a larger proportion of individuals with Lactobacillus -dominant urine, previous studies found that the UUI cohort exhibited decreasedLactobacillus sequence abundances compared to those for the control cohort, ${ }^{2}$ and in the $5 \mathrm{mg}$ Antimuscarinic responder group, Lactobacilluswas the most frequently cultivable urotype,${ }^{17}$ in contrast, women who developed a posttreatment UTI had fewerLactobacillus sequences. ${ }^{6}$ Lactobacillusis best known for its dominance in vaginal microbiota and it can prevent vaginitis by maintaining a physiological acidic environment in the vagina. It seems pretty clear thatLactobacillusin the bladder also plays an important regulatory or protective role in functional lower urinary tract (LUT) disorders.

The bacterial diversity (number of unique bacterial species) and richness were increased in the moderate/severe patients urinary microbiome, in contrast, the evenness, represents the distribution of microbial species within the sample, was not statistically different. Although the M group has a smaller sample size than the M/S group, we further found statistically significant trends in terms of species diversity and richness by socres of OABSS, and confirmed that the higher the socres the higher the Observed species, Chao1, ACE, and Shannon's index, verifying that the more severe the symptoms of OAB patients, the higher the diversity and richness of urine bacteria. This result is in consistent with the finding of PRICE ${ }^{16}$, but is inconsistent with the findings of Karstens L. ${ }^{5}$ PRICE et al. used an enhanced culture method called Expanded Quantitative Urine Culture (EQUC) coupled to MALDI-TOF mass spectrometry to identify the Chao1, ACE, and Shannon's Indices were positively correlated to Urinary Distress Inventory (UDI) subscale score, which measures the severity of the disease. ${ }^{16}$ But Karstens et al. found that lower microbial diversity was associated with increased symptom severity in women with UUI. ${ }^{5}$ We think this can be explained by the fact that two seemingly opposite structural changes in the bacterial flora are both destructive to the normal bacterial flora. 
Although little is known about the optimal characteristics of the urinary microbiome, extreme diversity is unlikely to be a preferred or advantageous biological state. The lowest diversity may be associated with a lack of beneficial microbes. At the other extreme, the increase in diversity may reflect the loss of the urinary flora regulation mechanism, which does not allow a healthy microbial community. Despite the differences in the composition of the inter-individual microbiota, individuals in the same group still have similarities in the urinary microbiota. as shown in the PCoA analysis that clustered mild group and moderate/severe group separately (Figure 2), indicating the possibility of a common urinary microbiota disorder associated with the same degree of OAB.

At phylum level, we detected that Actinobacteriaincreased significantly in the urine of moderate/severe patients, and our previous research found that compared with asymptomatic patients, OAB patients have significantly more Actinobacteria in their urine. ${ }^{8}$ These findings raise the possibility that dysbiotic microbial populations may play a role in the pathogenesis of OAB. At genus level, correlation analysis found that several specific bacteria are associated with different OAB sub-symptoms, some of which have also been reported by other researchers to be associated with LUTS. There was a positive correlation between abundance of Prevotella and the degree of Nighttime frequency. Coincidentally, in Pearce et al.'s study, ${ }^{2}$ they found that enrichment of Prevotella was observed in UUI patients. Emerging studies have linked the increased abundance of Prevotella species to localized disease, including periodontitis, bacterial vaginosis, rheumatoid arthritis, metabolic disorders and low-grade inflammation. ${ }^{18}$ Apostolidis A et al. found bladder biopsies in patients with $\mathrm{OAB}$ revealed signs of chronic inflammation. ${ }^{19}$ This suggests that the bladder microbiome may participate in the occurrence and development of LUTS by causing chronic inflammation. We also detected that the more severe the symptoms of Nighttime frequency, the higher abundance of Porphyromona. In addition, the same trend of Porphyromona abundance changes was observed in Shoskes et al.'study, which revealed that chronic prostatitis/chronic pelvic pain syndrome (CP/CPPS) patients had over-representation of genusPorphyromona ${ }^{20}$ All these indicate that the intriguing possibility that specific microbial patterns may be linked to specific symptoms, which provides a great enlightenment for future researches of the urinary microbiome and the algorithms for the diagnosis and treatment of LUTS..

\section{CONCLUSION}

In conclusion, findings in our study suggest that bladder microbiome is related to LUTS severity. The results of our research offer strong evidence that the higher bacterial diversity and richness was associated with worse OAB symptom severity, and several specific bacterial genera relate to sub-symptoms of OAB respectively, suggest that specific urinary dysbiosis may constitute an etiological factor in exacerbating functional bladder disorders, with potential diagnostic and therapeutic implications in the near future.

Disclosure: Nothing to disclose.

Funding: Supported by the National Natural Science Foundation of China (Grant No. 81870522).

Contribution to authorship: Conceptualization, Kun Li, Chunxiao Chen, Jie Zhao and Peng Wu; Patient recruitment, Specimens and demographic information collection, Kun Li, Chunxiao Chen, Jiarong Zeng, Yuehui Wen, Biao Li and Weihong Chen; Specimen processing and data analysis Jie Zhao and Peng Wu; Writing - original draft, Kun Li, Chunxiao Chen; Writing - review \& editing, Kun Li, Chunxiao Chen, Jie Zhao and Peng Wu.

\section{Details of ethics approval}

The study design was approved by Medical Ethics Committee of Nanfang Hospital, Southern Medical University (NFEC-2020-123 [28 May 2020]).

\section{Reference:}

1. Fouts DE, Pieper R, Szpakowski S, Pohl H, Knoblach S, Suh MJ, et al. Integrated next-generation sequencing of $16 \mathrm{~S}$ rDNA and metaproteomics differentiate the healthy urine microbiome from asymptomatic 
bacteriuria in neuropathic bladder associated with spinal cord injury. Journal of translational medicine. 2012 Aug 28;10:174.

2. Pearce MM, Hilt EE, Rosenfeld AB, Zilliox MJ, Thomas-White K, Fok C, et al. The female urinary microbiome: a comparison of women with and without urgency urinary incontinence. mBio. 2014 Jul 8;5(4):e01283-14.

3. Thomas-White KJ, Kliethermes S, Rickey L, Lukacz ES, Richter HE, Moalli P, et al. Evaluation of the urinary microbiota of women with uncomplicated stress urinary incontinence. American journal of obstetrics and gynecology. 2017 Jan;216(1):55.e1-.e16.

4. Abernethy MG, Rosenfeld A, White JR, Mueller MG, Lewicky-Gaupp C, Kenton K. Urinary Microbiome and Cytokine Levels in Women With Interstitial Cystitis. Obstetrics and gynecology. 2017 Mar;129(3):500-6.

5. Karstens L, Asquith M, Davin S, Stauffer P, Fair D, Gregory WT, et al. Does the Urinary Microbiome Play a Role in Urgency Urinary Incontinence and Its Severity? Frontiers in cellular and infection microbiology. 2016;6:78.

6. Pearce MM, Zilliox MJ, Rosenfeld AB, Thomas-White KJ, Richter HE, Nager CW, et al. The female urinary microbiome in urgency urinary incontinence. American journal of obstetrics and gynecology. 2015 Sep;213(3):347.e1-11.

7. Nickel JC, Stephens A, Landis JR, Mullins C, van Bokhoven A, Lucia MS, et al. Assessment of the Lower Urinary Tract Microbiota during Symptom Flare in Women with Urologic Chronic Pelvic Pain Syndrome: A MAPP Network Study. The Journal of urology. 2016 Feb;195(2):356-62.

8. Wu P, Chen Y, Zhao J, Zhang G, Chen J, Wang J, et al. Urinary Microbiome and Psychological Factors in Women with Overactive Bladder. Frontiers in cellular and infection microbiology. 2017;7:488.

9. An F, Yang X, Wang YJ, Chen JY, Wang JL. OAB epidemiological survey of general gynaecology outpatients and its effects on patient quality of life. Neurourology and urodynamics. 2016 Jan;35(1):29-35.

10. Haylen BT, de Ridder D, Freeman RM, Swift SE, Berghmans B, Lee J, et al. An International Urogynecological Association (IUGA)/International Continence Society (ICS) joint report on the terminology for female pelvic floor dysfunction. International urogynecology journal. 2010 Jan;21(1):5-26.

11. Segata N, Izard J, Waldron L, Gevers D, Miropolsky L, Garrett WS, et al. Metagenomic biomarker discovery and explanation. Genome biology. 2011 Jun 24;12(6):R60.

12. Wolfe AJ, Toh E, Shibata N, Rong R, Kenton K, Fitzgerald M, et al. Evidence of uncultivated bacteria in the adult female bladder. Journal of clinical microbiology. 2012 Apr;50(4):1376-83.

13. Hilt EE, McKinley K, Pearce MM, Rosenfeld AB, Zilliox MJ, Mueller ER, et al. Urine is not sterile: use of enhanced urine culture techniques to detect resident bacterial flora in the adult female bladder. Journal of clinical microbiology. 2014 Mar;52(3):871-6.

14. Khasriya R, Sathiananthamoorthy S, Ismail S, Kelsey M, Wilson M, Rohn JL, et al. Spectrum of bacterial colonization associated with urothelial cells from patients with chronic lower urinary tract symptoms. Journal of clinical microbiology. $2013 \mathrm{Jul} ; 51(7): 2054-62$.

15. Price TK, Dune T, Hilt EE, Thomas-White KJ, Kliethermes S, Brincat C, et al. The Clinical Urine Culture: Enhanced Techniques Improve Detection of Clinically Relevant Microorganisms. Journal of clinical microbiology. 2016 May;54(5):1216-22.

16. Price TK, Lin H, Gao X, Thomas-White KJ, Hilt EE, Mueller ER, et al. Bladder bacterial diversity differs in continent and incontinent women: a cross-sectional study. American journal of obstetrics and gynecology. 2020 May 5. 
17. Thomas-White KJ, Hilt EE, Fok C, Pearce MM, Mueller ER, Kliethermes S, et al. Incontinence medication response relates to the female urinary microbiota. International urogynecology journal. 2016 May;27(5):723-33.

18. Larsen JM. The immune response to Prevotella bacteria in chronic inflammatory disease. Immunology. 2017 Aug;151(4):363-74.

19. Apostolidis A, Jacques TS, Freeman A, Kalsi V, Popat R, Gonzales G, et al. Histological changes in the urothelium and suburothelium of human overactive bladder following intradetrusor injections of botulinum neurotoxin type A for the treatment of neurogenic or idiopathic detrusor overactivity. European urology. 2008 Jun;53(6):1245-53.

20. Shoskes DA, Altemus J, Polackwich AS, Tucky B, Wang H, Eng C. The Urinary Microbiome Differs Significantly Between Patients With Chronic Prostatitis/Chronic Pelvic Pain Syndrome and Controls as Well as Between Patients With Different Clinical Phenotypes. Urology. 2016 Jun;92:26-32.

\section{Hosted file}

Table.docx available at https://authorea.com/users/356405/articles/479269-interplay-betweenbladder-microbiota-and-overactive-bladder-symptom-severity-a-cross-sectional-study

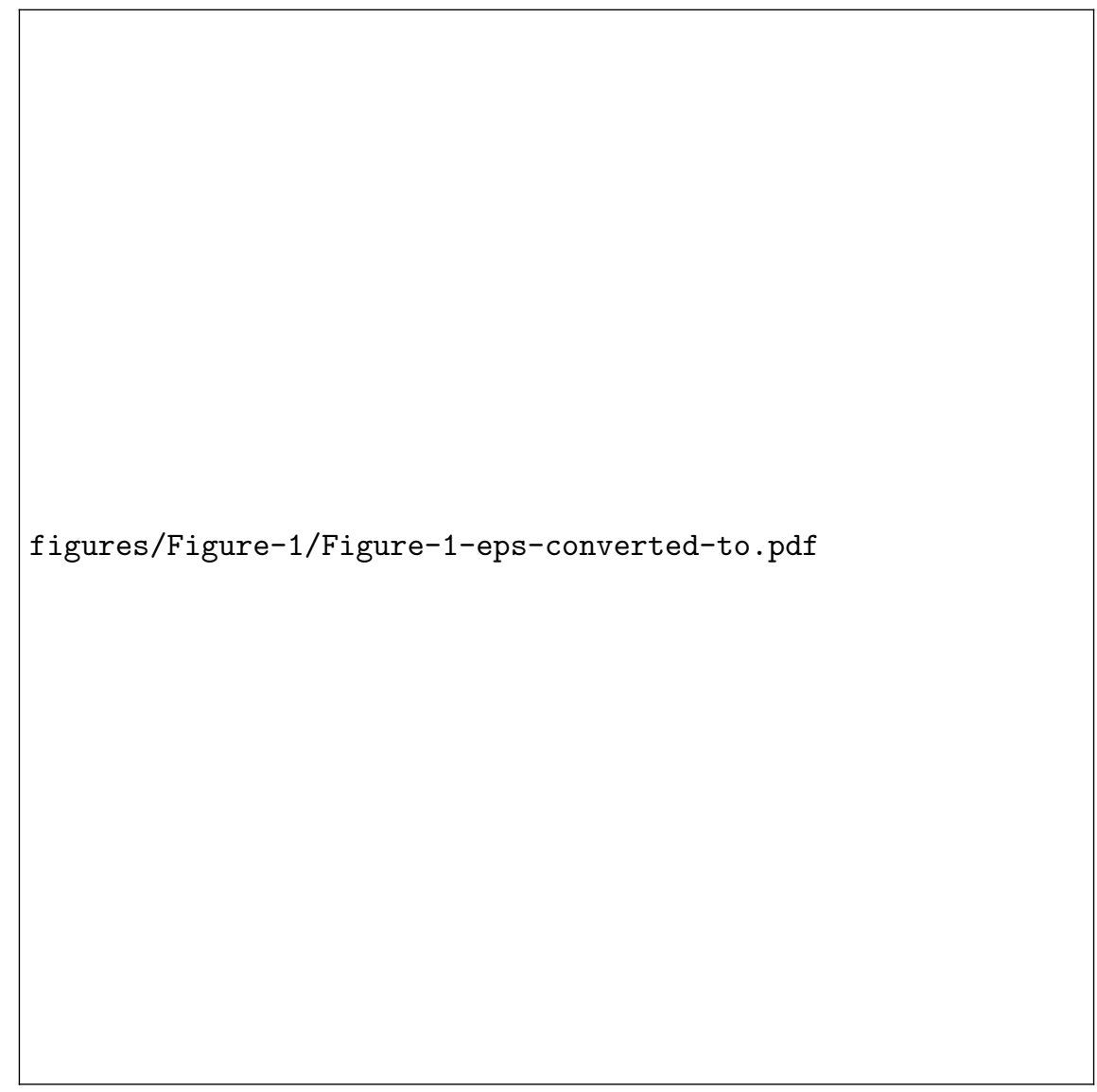


figures/Figure-2/Figure-2-eps-converted-to.pdf 